\title{
Lack of Association between Genetic Polymorphisms of JAK-STAT Signaling Pathway Genes and Acute Anterior Uveitis in Han Chinese
}

\author{
Ling Cheng, Hongsong Yu, Yan Jiang, Juan He, Sisi Pu, Xin Li, and Li Zhang \\ The First Affiliated Hospital of Chongqing Medical University, Chongqing Key Laboratory of Ophthalmology and \\ Chongqing Eye Institute, Chongqing, China \\ Correspondence should be addressed to Li Zhang; zhangli298@hotmail.com
}

Received 2 June 2016; Revised 14 September 2016; Accepted 16 October 2016

Academic Editor: Juan-Manuel Anaya

Copyright ( 2016 Ling Cheng et al. This is an open access article distributed under the Creative Commons Attribution License, which permits unrestricted use, distribution, and reproduction in any medium, provided the original work is properly cited.

\begin{abstract}
Purpose. This study aimed to investigate the association between single nucleotide polymorphisms (SNPs) of JAK-STAT signaling pathway genes and acute anterior uveitis (AAU) with or without ankylosing spondylitis (AS) in the Han Chinese population. Methods. Eleven SNPs of the JAK1, JAK2, STAT1, IRF1, and NOS2 genes were analyzed in 443 AAU patients with AS, 486 AAU patients without AS, and 714 healthy controls. Genotyping was performed by PCR-RFLP assay or TaqMan ${ }^{\circledR}$ probe assay. The Chisquared $\left(\chi^{2}\right)$ test and multivariate logistic regression analysis were used to compare the distributions of alleles and genotypes between patients and controls. $P$ values were adjusted using Bonferroni correction. Results. We did not observe significant differences in the genotype and allele frequencies of any SNP between AAU patients with or without AS and healthy controls. Stratification analyses by gender and HLA-B27 status showed a boundary significant association between two SNPs (rs10975003 and rs10758669) in JAK2 and AAU $(P=0.052$ and $P=0.053$, resp.). Conclusions. Our results indicated that genetic polymorphisms of the JAK-STAT signaling pathway genes may not be associated with AAU in the Han Chinese population.
\end{abstract}

\section{Introduction}

Uveitis is one of the major ocular diseases leading to blindness and visual impairment. The prevalence of uveitis is 111.3 per 100,000 persons in Taiwan [1] compared with 40.4 per 100,000 persons in Japan [2] and 115.3 per 100,000 persons in United States [3]. In the clinic, acute anterior uveitis (AAU), which may be accompanied by complicated phenotypes including cataract and glaucoma [4], is the most common type of uveitis [5]. Evidence suggests that the occurrence of AAU is associated with the prognosis of ankylosing spondylitis (AS) $[6,7]$. The frequency of AAU, which is characterized by positive human leukocyte antigen- (HLA-) $B 27$, varies across different ethnic populations [8-10]. In the United States and Western Europe, the prevalence of HLAB27 with AAU is up to $50 \%[5,8,11]$. Previous studies have reported that there is a strong association between AS and HLA-B27 in various ethnic groups [12-14]. Further study showed that the percentage of AAU accompanied by AS is
$30-40 \%$, suggesting that there may be linked pathogenesis between AAU and AS [15]. AAU and AS may share certain genetic associations, but several susceptibility genes seem to be unique for each disease [16]. Genes including TNFSF15, TRAF5, and FoxO1 have been reported to be associated with AAU [17-19]. However, a lack of association with AAU has been demonstrated for other genes, including CTLA4 and PTPN22 [20, 21]. A recent study revealed that T lymphocyte subsets (Th1 and Th17) and $\mathrm{CD}^{+}{ }^{+} \mathrm{CD} 25^{+}$Treg cells were involved in the development of HLA-B27 positive AAU $[22,23]$. Furthermore, a higher level of Th17 cells has been observed in the peripheral blood of patients with AS [24].

The Janus kinase-signal transducer and activator of transcription (JAK-STAT) signaling pathway plays a major role in T lymphocyte differentiation and function $[25,26]$. JAK1 and JAK2 have been reported to play an important role in Th1 and Th17 cell differentiation [27]. STAT1 is critical to T lymphocyte differentiation and function [25, 26, 28]. STAT1 is activated by type I interferons (IFNs) and IFN- $\gamma$ and plays 
an important role in immune responses [29]. IRF1 is the first member identified in the IRF family and is involved in many innate and adaptive immune responses [30]. Impaired or absent Th1-type immune responses favor Th2 differentiation in IRF1-deficient mice [31,32]. NOS2-derived NO, a key factor in immunoregulation [33], can inhibit Th1 as well as Th2 cytokine production and regulate the development of FoxP3 ${ }^{+}$ Treg cells $[34,35]$. In summary, JAK-STAT signaling pathway genes, including JAK1, JAK2, STAT1, IRF1, and NOS2, have been suggested to be strongly linked with $T$ cells and may be involved in the pathophysiology of AAU with or without AS.

Thus, we conducted the present case-control study to investigate whether JAK-STAT signaling pathway genes confer susceptibility to AAU risk in a Chinese Han population.

\section{Materials and Methods}

2.1. Subjects. A total of 929 AAU patients were enrolled in this study, including 443 patients with $\mathrm{AS}\left(\mathrm{AAU}^{+} \mathrm{AS}^{+}\right)$and 486 patients without $\mathrm{AS}\left(\mathrm{AAU}^{+} \mathrm{AS}^{-}\right)$, as well as 714 genderand race-matched healthy controls. All subjects were Han Chinese recruited from the Department of Ophthalmology in the First Affiliated Hospital of Chongqing Medical University (Chongqing, China) between June 2008 and May 2015. All AAU patients were diagnosed based on medical records, physical examinations, and the anatomic location of inflammation as previously described by Jabs et al. [36]. The diagnosis of AS followed the modified New York Criteria [37]. All subjects gave written informed consent before blood collection. This study was approved by the Human Ethics Committee of the First Affiliated Hospital of Chongqing Medical University (Approval number: 2009-201008) and followed the tenets of the Declaration of Helsinki.

2.2. SNP Selection. We selected candidate single nucleotide polymorphisms (SNPs) based on previously published studies and included only those SNPs significantly associated with autoimmune diseases [38-45]. We used HaploView 4.2 software to evaluate the linkage disequilibrium (LD) and minor allele frequency (MAF) of the SNPs. Five SNPs of $J A K 1, \mathrm{rs} 2780815$, rs3790532, rs310230, rs310236, and rs310241 $[41,42]$, were selected. Since the SNPs rs3790532, rs310230, rs310236, and rs310241 are in strong LD with each other $\left(r^{2}>\right.$ 0.8 , Figure 1), we only used rs310241 in our study. Furthermore, we also eliminated SNPs that were not polymorphic in the Chinese population. Finally, eleven SNPs in five JAKSTAT signaling pathway genes were tested in our study, including two SNPs in the intron region of the JAK1 gene (rs310241, rs2780815) [41], two SNPs in the exon region and $3^{\prime} \mathrm{UTR}$ of the JAK2 gene (rs10758669, rs10975003) [38, 45], one SNP in the intron region of the IRF1 gene (rs2070721), four SNPs in the exon region and intron region of the STAT1 gene (rs2066802, rs1547550, rs6718902, and rs10199181) [39, $40]$, and two SNPs in the exon region and intron region of the NOS2 gene (rs2297518, rs4795067) [43, 44].

2.3. DNA Extraction and Genotyping. Peripheral blood samples were collected from subjects, and genomic DNA extraction was performed using the QIAamp DNA Blood Mini
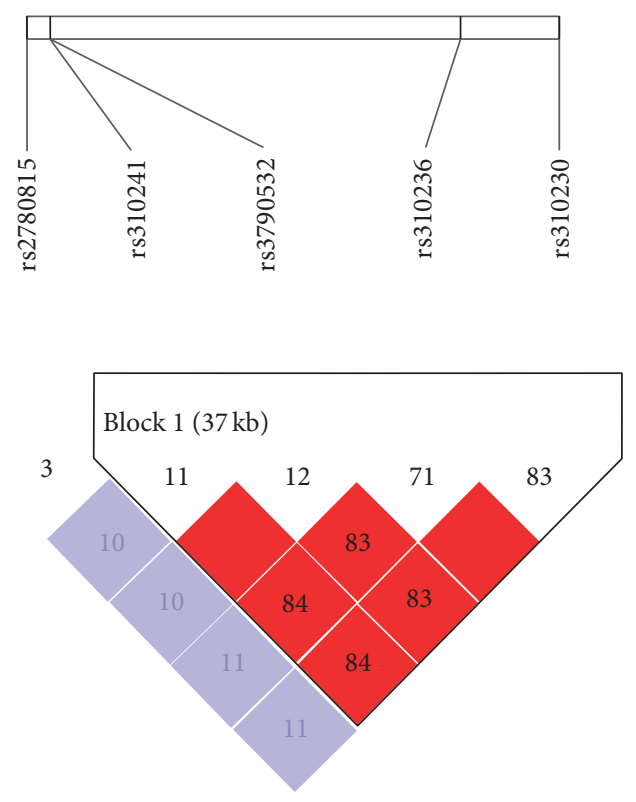

FIGURE 1: Linkage disequilibrium (LD) analysis of rs2780815, rs310241, rs3790532, rs310236, and rs310230 in the JAK1 gene. The LD block was estimated by HaploView software version 4.2 using the Chinese Han HapMap data. The number in the square indicates the $r^{2}$ value.

Kit (Qiagen, Valencia, CA, USA). The genomic DNA was quantified with NanoDrop 2000 (Thermal Fisher Scientific, Delaware, USA) and stored at $-20^{\circ} \mathrm{C}$ until use. Three SNPs (rs2780815, rs2070721, and rs10199181) were genotyped by TaqMan probe (Applied Biosystems, Foster City, CA), and the others were genotyped by polymerase chain reaction restriction fragment length polymorphism (PCR-RFLP) assay. The specific primers for PCR and restriction enzymes are described in Table 1 . The PCR reactions were performed under the following conditions: denaturation at $95^{\circ} \mathrm{C}$ for 5 minutes, 33 to 36 cycles of denaturation at $95^{\circ} \mathrm{C}$ for 30 seconds, annealing at $56-64^{\circ} \mathrm{C}$ for $30 \mathrm{~s}$, and extension at $72^{\circ} \mathrm{C}$ for 30 seconds and a final extension at $72^{\circ} \mathrm{C}$ for 5 minutes. The enzyme-digested products were visualized on $3 \%$ or $4 \%$ agarose gels and stained with GoldView (SBS Genetech, Beijing, China). Direct sequencing was carried out randomly on $10 \%$ of the study samples to assure the validity of the SNP genotyping method used. The success rate of all SNP genotyping ranged from $97.3 \%$ to $100 \%$.

2.4. Statistical Analysis. Hardy-Weinberg equilibrium (HWE) was analyzed by the $\chi^{2}$ test. The distributions of the allele and genotype frequencies between the patients and controls were compared by the $\chi^{2}$ test. Multivariate logistic regression model adjusted for age and gender was further adopted to test the associations between the SNPs and AAU. The risk effect of each SNP was measured by odds ratios (OR) and $95 \%$ confidence intervals (CI). There were four different models of inheritance in our study, including additive models, codominant models, dominant models, and recessive models. $P$ values were corrected using the Bonferroni correction 
TABLE 1: PCR primer sequences and restriction enzymes.

\begin{tabular}{llc}
\hline SNP & Primers & Restriction enzyme \\
\hline rs310241 & 5' AACCACCAGCTCAACATTCCTAG 3' & BseDI \\
rs6718902 & 5' CAGCCAGGTCTCCCGTAGG 3' & DraI \\
rs2297518 & 5' CGGACAAAAGCATGCACTAGA 3' & TaqI \\
rs10758669 & 5' CCACCACCATTAATAGGTGACTTTA 3' & BanI \\
rs10975003 & 5' TGAGCTCTTTCAGCATGAAGATC 3' & HpaI \\
rs4795067 & 5' CTTCCGTGGTGGGCTGTG 3' & NdeI \\
rs1547550 & 5' TGATGTAGAGACAAGGACATGCTGAGGTAC 3' & \\
rs2066802 & 5' GCCAAAAGACAAAGGCAAGGGG 3' & BstMAI \\
& 5' GGCCAGTCAAGAAAAAACCAGTT 3' & 5indIII \\
\hline
\end{tabular}

method considering multiple tests. Statistical significance level was defined as a corrected $P$ value $<0.05$. Statistical analyses were performed using SPSS version 17.0 (SPSS, Inc., Chicago, IL, USA).

\section{Results}

3.1. Clinical Features of AAU Patients. The detailed clinical features and demographic characteristics of the AAU patients are presented in Table 2. All 929 AAU patients include 569 $(61.2 \%)$ males and $360(38.8 \%)$ females. The 714 control subjects consisted of 428 (59.9\%) males and $286(40.1 \%)$ females. The average age was $39.8 \pm 12.3$ in AAU patients and $39.5 \pm 10.8$ in the controls, respectively. There were no significant differences in age and gender between the cases and controls. In addition, 546 AAU patients (68.9\%) were HLA-B27-positive, whereas 246 AAU patients (31.1\%) were HLA-B27-negative.

3.2. The Genotype and Allele Frequency Distribution of the Tested SNPs in AAU. Eleven SNPs of the JAK-STAT signaling pathway genes (JAK1, JAK2, STAT1, IRF1, and NOS2) were successfully genotyped. There were no significant deviations of HWE in either the cases or controls. We did not observe significant differences in the genotype and allele distributions of any of the SNPs between the AAU patients and control subjects after Bonferroni correction (see Supplementary Table S1 in Supplementary Material available online at http://dx.doi.org/10.1155/2016/5896906). Further stratified analyses of gender, AS, and HLA-B27 status showed a boundary significant association of two SNPs (rs10975003 and rs10758669) of JAK2 with AAU. In female AS-positive AAU patients, there was a decreased frequency of the TT genotype of rs10975003 compared to the female controls $(\mathrm{OR}=0.55$; $P=1.59 \times 10^{-3} ; P_{\text {Bonferroni }}=0.052$, Table 3$)$, whereas no significant differences were observed in the genotype and allele frequencies of the other ten SNPs $\left(P_{\text {Bonferroni }}>0.05\right.$, Table 3). Similarly, there were no significant differences in
TABLE 2: Clinical characteristics of the investigated subjects.

\begin{tabular}{lcc}
\hline Clinical features & Total & Percentage \\
\hline AAU patients & 929 & 100 \\
Mean age \pm SD (years) & $39.8 \pm 12.3$ & \\
AAU with AS & 443 & 47.7 \\
AAU without AS & 486 & 52.3 \\
AAU male & 569 & 61.2 \\
AAU female & 360 & 38.8 \\
AAU with AS (male) & $326(443$ tested) & 73.6 \\
AAU with AS (female) & $117(443$ tested) & 26.4 \\
AAU without AS (male) & $243(486$ tested) & 50 \\
AAU without AS (female) & $243(486$ tested) & 50 \\
HLA-B27 $7^{+}$AAU & $546(792$ tested) & 68.9 \\
HLA-B27 $7^{+}$AAU AS ${ }^{+}$ & $348(428$ tested) & 81.3 \\
HLA-B27 $7^{+}$AAU AS & $198(364$ tested) & 54.4 \\
Control & 714 & 100 \\
Mean age \pm SD (years) & $39.5 \pm 10.8$ & \\
Male & 428 & 59.9 \\
Female & 286 & 40.1 \\
\hline
\end{tabular}

the genotype and allele frequencies of the SNPs between the male AAU patients and male controls $\left(P_{\text {Bonferroni }}>0.05\right.$, Supplementary Table S2).

In addition, an increased frequency of the AC genotype in rs10758669 was observed in HLA-B27-positive AAU patients compared to the healthy controls $\left(\mathrm{OR}=1.44 ; P=1.62 \times 10^{-3}\right.$; $P_{\text {Bonferroni }}=0.053$, Table 4$)$, whereas there were no significant differences in the genotype and allele frequencies of the other ten SNPs between the HLA-B27-positive AAU patients and the control subjects $\left(P_{\text {Bonferroni }}>0.05\right.$, Table 4$)$.

However, an increased frequency of the rs10758669/AC genotype was observed in HLA-B27-positive AS-positive AAU patients compared to healthy controls $(\mathrm{OR}=1.49 ; P=$ $2.56 \times 10^{-3} ; P_{\text {Bonferroni }}=0.084$, Table 5), whereas no significant 


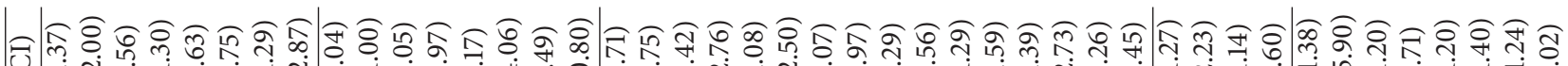

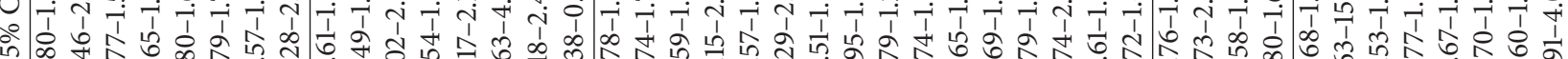

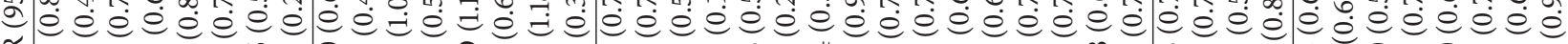
б

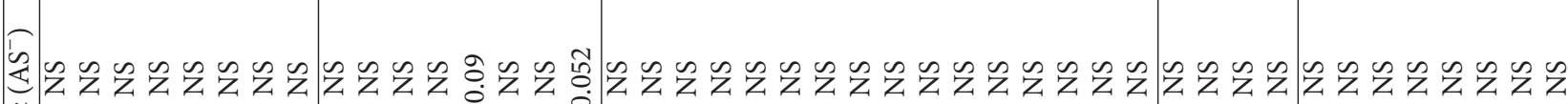
茨

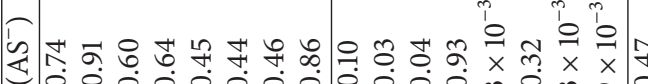

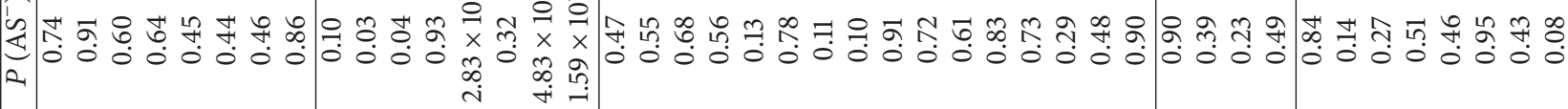

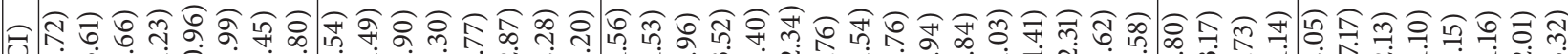

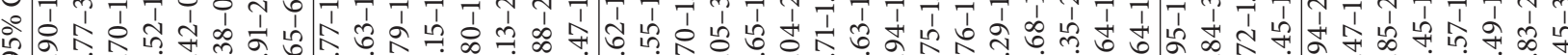

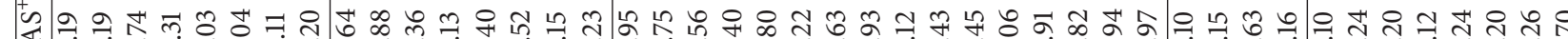

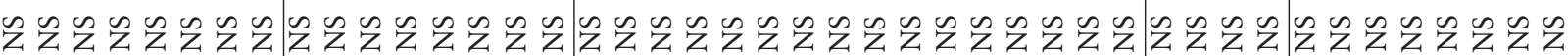

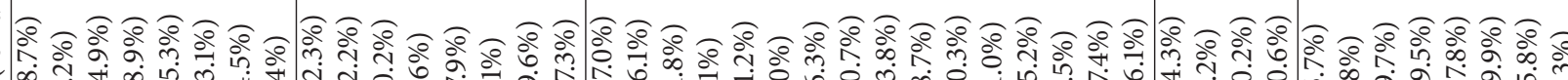
क्⿹

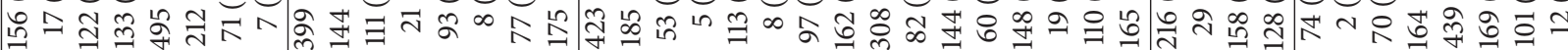

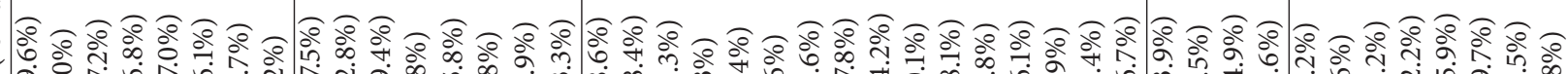

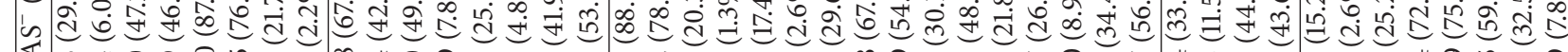

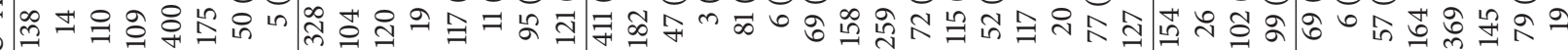

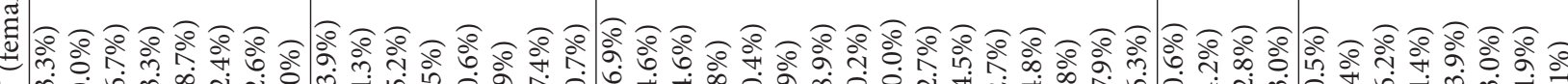

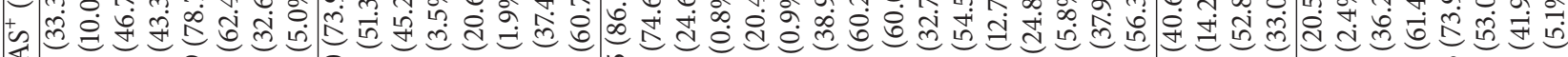

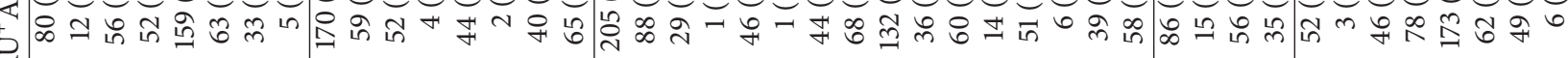

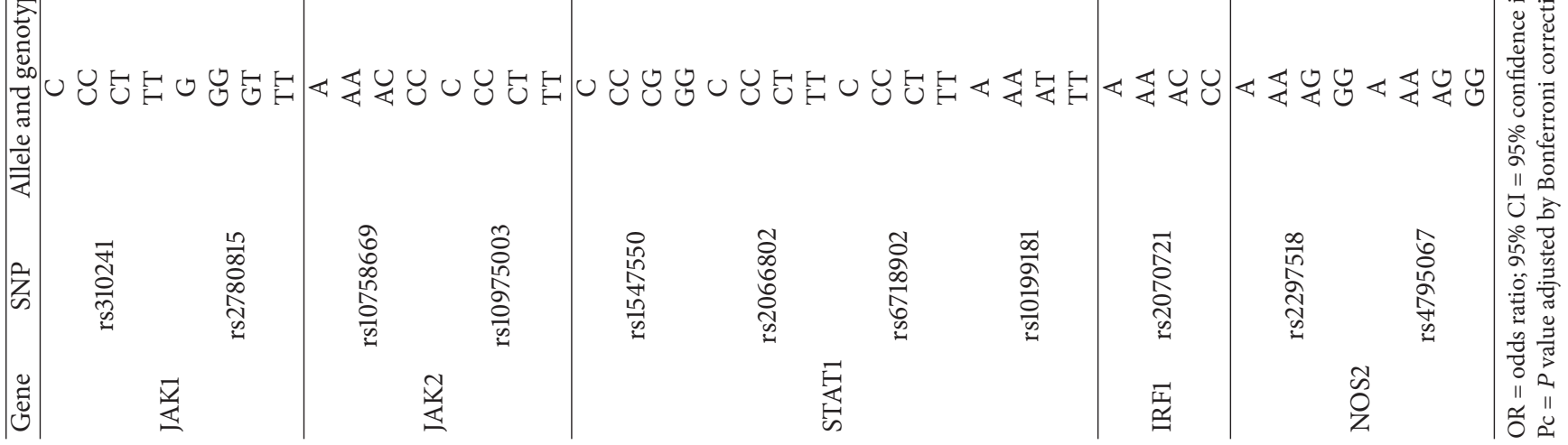


TABLE 4: Allele and genotype frequencies of SNPs in patients with AAU versus control subjects stratified by HLA-B27 status.

\begin{tabular}{|c|c|c|c|c|c|c|c|}
\hline Gene & SNP & Allele and genotype & $\begin{array}{c}\text { AAU } \\
\text { HLA-B27 }\end{array}$ & Control & $P$ & $\mathrm{Pc}$ & OR (95\% CI) \\
\hline \multirow{8}{*}{ JAK1 } & \multirow{3}{*}{ rs310241 } & $\mathrm{C}$ & $324(31.0 \%)$ & $392(27.5 \%)$ & 0.06 & NS & $1.18(1.00-1.41)$ \\
\hline & & $\mathrm{CC}$ & $41(7.9 \%)$ & $45(6.3 \%)$ & 0.29 & NS & $1.27(0.82-1.96)$ \\
\hline & & $\mathrm{CT}$ & $242(46.4 \%)$ & $302(42.4 \%)$ & 0.16 & NS & $1.18(0.94-1.48)$ \\
\hline & \multirow{5}{*}{ rs2780815 } & $\mathrm{TT}$ & 239 (45.7\%) & $366(51.3 \%)$ & 0.05 & NS & $0.80(0.64-1.00)$ \\
\hline & & $\mathrm{G}$ & $878(86.2 \%)$ & $1250(87.5 \%)$ & 0.35 & NS & $0.89(0.70-1.13)$ \\
\hline & & GG & $381(74.8 \%)$ & $548(76.7 \%)$ & 0.44 & NS & $0.90(0.69-1.18)$ \\
\hline & & GT & $116(22.8 \%)$ & $154(21.6 \%)$ & 0.61 & NS & $1.07(0.82-1.41)$ \\
\hline & & $\mathrm{TT}$ & $12(2.4 \%)$ & $12(1.7 \%)$ & 0.40 & NS & $1.41(0.63-3.17)$ \\
\hline \multirow{8}{*}{ JAK2 } & \multirow{3}{*}{ rs10758669 } & $\mathrm{A}$ & $705(65.9 \%)$ & $980(69.0 \%)$ & 0.10 & NS & $0.87(0.73-1.03)$ \\
\hline & & $\mathrm{AA}$ & $220(41.1 \%)$ & $346(48.7 \%)$ & 0.08 & NS & $0.74(0.59-0.92)$ \\
\hline & & $\mathrm{AC}$ & $265(49.5 \%)$ & $288(40.6 \%)$ & $1.62 \times 10^{-3}$ & 0.053 & $1.44(1.15-1.80)$ \\
\hline & \multirow{5}{*}{ rs10975003 } & $\mathrm{CC}$ & $50(9.4 \%)$ & 76 (10.7\%) & 0.43 & NS & $0.86(0.59-1.25)$ \\
\hline & & $\mathrm{C}$ & $251(24.5 \%)$ & $272(21.2 \%)$ & 0.05 & NS & $1.21(1.00-1.47)$ \\
\hline & & $\mathrm{CC}$ & $26(5.1 \%)$ & $28(4.4 \%)$ & 0.56 & NS & $1.18(0.68-2.03)$ \\
\hline & & $\mathrm{CT}$ & $199(38.8 \%)$ & $216(33.5 \%)$ & 0.06 & NS & $1.26(0.99-1.60)$ \\
\hline & & $\mathrm{TT}$ & $287(56.1 \%)$ & $399(62.1 \%)$ & 0.03 & NS & $0.77(0.61-0.98)$ \\
\hline \multirow{16}{*}{ STAT1 } & \multirow{4}{*}{ rs1547550 } & $\mathrm{C}$ & $937(87.2 \%)$ & $1254(87.8 \%)$ & 0.67 & NS & $0.95(0.75-1.21)$ \\
\hline & & $\mathrm{CC}$ & $407(75.8 \%)$ & 555 (77.7\%) & 0.42 & NS & $0.90(0.69-1.17)$ \\
\hline & & CG & $123(22.9 \%)$ & $144(20.2 \%)$ & 0.24 & NS & $1.18(0.90-1.54)$ \\
\hline & & GG & $7(1.3 \%)$ & $15(2.1 \%)$ & 0.29 & NS & $0.62(0.25-1.52)$ \\
\hline & \multirow{4}{*}{ rs2066802 } & $\mathrm{C}$ & $190(19.3 \%)$ & $288(20.7 \%)$ & 0.39 & NS & $0.91(0.74-1.12)$ \\
\hline & & $\mathrm{CC}$ & $15(3.0 \%)$ & 27 (3.9\%) & 0.44 & NS & $0.78(0.41-1.48)$ \\
\hline & & $\mathrm{CT}$ & $160(32.5 \%)$ & $234(33.7 \%)$ & 0.66 & NS & $0.95(0.74-1.21)$ \\
\hline & & $\mathrm{TT}$ & $318(64.5 \%)$ & $434(62.4 \%)$ & 0.47 & NS & $1.09(0.86-1.39)$ \\
\hline & \multirow{4}{*}{ rs6718902 } & $\mathrm{C}$ & $584(56.5 \%)$ & $778(54.5 \%)$ & 0.33 & NS & $1.08(0.92-1.27)$ \\
\hline & & $\mathrm{CC}$ & $163(31.5 \%)$ & $209(29.3 \%)$ & 0.40 & NS & $1.11(0.87-1.42)$ \\
\hline & & $\mathrm{CT}$ & 258 (49.9\%) & $360(50.4 \%)$ & 0.86 & NS & $0.98(0.78-1.23)$ \\
\hline & & $\mathrm{TT}$ & $96(18.6 \%)$ & 145 (20.3\%) & 0.45 & NS & $0.90(0.67-1.19)$ \\
\hline & \multirow{4}{*}{ rs10199181 } & A & $270(27.2 \%)$ & 397 (27.8\%) & 0.75 & NS & $0.97(0.81-1.16)$ \\
\hline & & AA & $43(8.7 \%)$ & $55(7.7 \%)$ & 0.55 & NS & $1.14(0.75-1.73)$ \\
\hline & & $\mathrm{AT}$ & $184(37.1 \%)$ & $287(40.2 \%)$ & 0.28 & NS & $0.88(0.69-1.11)$ \\
\hline & & $\mathrm{TT}$ & $269(54.2 \%)$ & $372(52.1 \%)$ & 0.47 & NS & $1.09(0.87-1.37)$ \\
\hline \multirow{4}{*}{ IRF1 } & \multirow{4}{*}{ rs2070721 } & $\mathrm{A}$ & $356(35.7 \%)$ & $487(34.1 \%)$ & 0.40 & NS & $1.08(0.91-1.27)$ \\
\hline & & AA & $61(12.2 \%)$ & $74(10.4 \%)$ & 0.30 & NS & $1.21(0.84-1.73)$ \\
\hline & & $\mathrm{AC}$ & $234(47.0 \%)$ & $339(47.4 \%)$ & 0.87 & NS & $0.98(0.78-1.23)$ \\
\hline & & $\mathrm{CC}$ & $203(40.8 \%)$ & $301(42.2 \%)$ & 0.63 & NS & $0.94(0.75-1.19)$ \\
\hline \multirow{8}{*}{ NOS2 } & \multirow{3}{*}{ rs2297518 } & $\mathrm{A}$ & $194(18.1 \%)$ & $209(16.2 \%)$ & 0.21 & NS & $1.15(0.93-1.42)$ \\
\hline & & AA & $12(2.3 \%)$ & $12(1.9 \%)$ & 0.64 & NS & $1.21(0.54-2.72)$ \\
\hline & & $\mathrm{AG}$ & $170(31.7 \%)$ & $185(28.6 \%)$ & 0.24 & NS & $1.16(0.90-1.49)$ \\
\hline & \multirow{5}{*}{ rs4795067 } & GG & $354(66.0 \%)$ & $450(69.5 \%)$ & 0.20 & NS & $0.85(0.67-1.09)$ \\
\hline & & A & $797(74.3 \%)$ & $1008(76.1 \%)$ & 0.35 & NS & $0.92(0.76-1.10)$ \\
\hline & & $\mathrm{AA}$ & $293(54.6 \%)$ & $382(57.7 \%)$ & 0.33 & NS & $0.89(0.71-1.23)$ \\
\hline & & $\mathrm{AG}$ & $211(39.4 \%)$ & $244(36.9 \%)$ & 0.37 & NS & $1.11(0.88-1.41)$ \\
\hline & & GG & $32(6.0 \%)$ & $36(5.4 \%)$ & 0.69 & NS & $1.10(0.68-1.80)$ \\
\hline
\end{tabular}

OR $=$ odds ratio; $95 \% \mathrm{CI}=95 \%$ confidence interval.

$\mathrm{Pc}=P$ value adjusted by Bonferroni correction. 


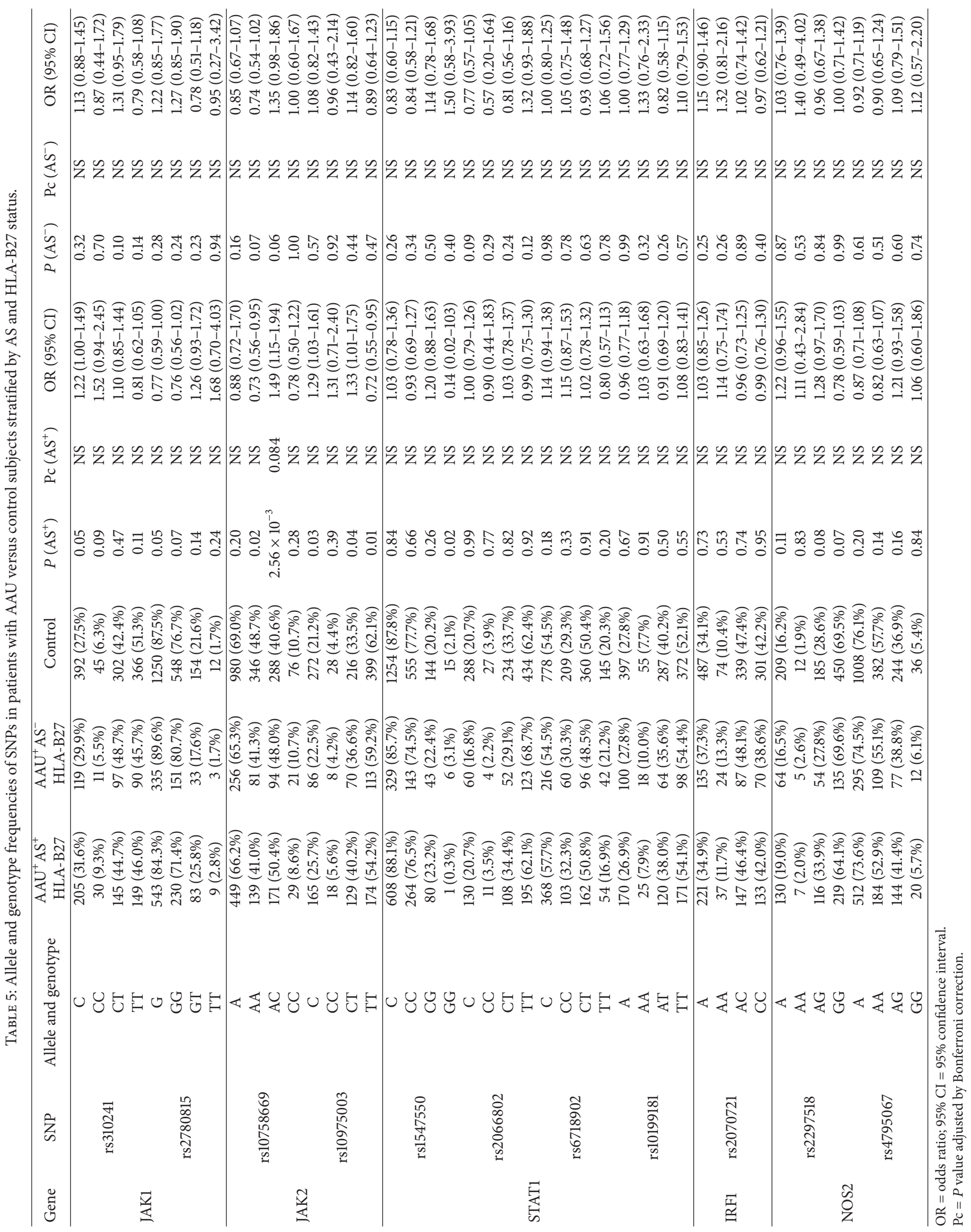


differences in the genotype and allele frequencies of the other 10 SNPs were observed between the HLA-B27-positive ASpositive AAU patients and healthy controls $\left(P_{\text {Bonferroni }}>0.05\right.$, Table 5). In addition, there were no significant differences in the genotype and allele frequencies of the tested SNPs between the AS-positive AAU patients and control subjects $\left(P_{\text {Bonferroni }}>0.05\right.$, Supplementary Table S3).

3.3. Logistic Regression Analysis of SNPs in AAU. We further investigated the SNPs rs10758669 and rs10975003 using additive, codominant, dominant, and recessive genetic models using a multivariate logistic regression model adjusted for age and gender. We observed that the frequency of the CA genotype of rs10758669 was significantly higher in AAU patients, which suggests that patients with the rs10758669 CA genotype have increased susceptibility to AAU $(46.8 \%$ versus $40.6 \%$, OR $=1.28, P=0.02$, Supplementary Table S4). An increased frequency of the AC genotype of rs10758669 was also observed in HLA-B27-positive AAU patients and AS-positive AAU patients compared to the healthy controls $(49.5 \%$ versus $40.6 \%$, OR $=1.43, P=$ $3.50 \times 10^{-3}$ and $49.2 \%$ versus $40.6 \%, \mathrm{OR}=1.36, P=0.02$, Supplementary Table S4). For SNP rs10975003, the frequency of the heterozygous CT genotype was significantly higher in female AAU patients compared to the healthy controls (40.4\% versus $33.6 \%$, OR $=1.57, P=0.01$, Supplementary Table S5). A similar result was observed when we combined CT and CC to construct a dominant model (44.3\% versus $38.0 \%, \mathrm{OR}=1.57, P=9.10 \times 10^{-3}$, Supplementary Table S5). However, none of the observed associations for the two SNPs retained statistical significance after Bonferroni correction $\left(P_{\text {Bonferroni }}>0.05\right)$. Furthermore, no significance associations were found between the other SNPs and AAU, even after stratification by gender, AS, and HLA-B27 status (data not shown).

\section{Discussion}

In this study, we first investigated whether genetic polymorphisms of JAK-STAT signaling pathway genes, including JAK1, JAK2, STAT1, IRF1, and NOS2, confer susceptibility to AAU with or without AS in a Chinese Han population. Our results suggest that none of these SNPs exhibit statistically different frequencies of genotypes and alleles between healthy controls and AAU patients. However, we observed a boundary significant association for two SNPs (rs10975003 and rs10758669) of JAK2 by stratification analysis by gender and HLA-B27 status.

We highlighted two issues at the time of study design to obtain unbiased association results. First, we followed strict criteria for the diagnosis of AAU patients. Patients with AAU were diagnosed as previously described by Jabs et al. [36] and patients with AS were diagnosed with the modified New York Criteria [37]. In addition, the AAU patients and healthy controls were strictly matched by ethnicity and age to avoid a possible influence of population stratification. Furthermore, we only enrolled controls who had detailed histories and physical examinations and excluded controls with any autoimmune or immune-related diseases. Finally,
$20 \%$ of the samples were randomly chosen and analyzed by direct sequencing, and the results of different genotyping methods were consistent.

AAU is defined as inflammation confined to the anterior segment of the eye that involves the iris and anterior part of the ciliary body. The HLA-B27 is considered to be strongly associated with both AAU and AS [5, 8, 10, 14]. Recent genetic studies have revealed that five candidate genes in the JAK-STAT signaling pathway were considered genetic predisposing factors for different autoimmune-mediated diseases [38-45]. SNPs (rs10758669 and 10975003) in JAK2 are considered susceptibility factors for Crohn's disease (CD) in the German population and ulcerative colitis (UC) in the Korean population $[38,45]$. One SNP (rs2070721) in IRF1 and SNPs (rs2297518 and rs4795067) in NOS2 are also associated with autoimmune diseases, such as multiple sclerosis (MS) in Italy, AS in Europe, and psoriasis in Pakistan [39, 43, 44]. Four SNPs (rs6718902, rs10199181, rs2066802, and rs1547550) in the STAT1 gene were observed to be associated with MS in Italy and $\operatorname{IgA}$ nephropathy $(\operatorname{IgAN})$ in Korea $[39,40]$. In addition, an association was found between SNPs (rs2780815, rs310241, rs3790532, rs310230, and rs310236) in JAK1 and Behçet disease (BD) as well as Vogt-Koyanagi-Harada (VKH) syndrome [41, 42], two other common uveitis entities in China. There has been no report of associations between JAK-STAT signaling pathway genes and AAU, and thus we performed this case-control study to detect whether the five candidate genes were associated with AAU in a Chinese Han population. Our results showed that there were no significant associations between the genetic polymorphisms of the five candidate genes in the JAK-STAT signaling pathway and AAU. These results are not consistent with those observed for other autoimmune diseases reported in German, European, and some other Asian populations [38, 43, 45]. This discrepancy may be attributable to differences in the etiology and pathogenesis of AAU compared with $\mathrm{BD}, \mathrm{VKH}$ syndrome, and autoimmune-mediated diseases.

Consistent with our results, a recent study also reported no significant association of JAK-STAT signaling pathway gene polymorphisms with rheumatoid arthritis stratified by the presence/absence of cardiovascular disease [46]. Conversely, an influence of NFKB1 signaling pathway polymorphisms on the development of cardiovascular events in patients with rheumatoid arthritis has been observed [47]. Furthermore, NFKB1 signaling pathway polymorphisms have been described to play a critical role in the development of many autoimmune and inflammatory diseases, and thus the evaluation of the potential relationships between NFKB1 polymorphisms and the development of AAU could be a promising research line for the future.

There were several limitations of our study. We had a limited sample size to detect SNPs with weak effects while considering multiple corrections. Even for SNPs rs10758669 and rs 10975003 , we only had $70.0 \%$ power using a genetic power calculator [48]. In addition, our samples were restricted to the Han Chinese population, and all patients were enrolled from the ophthalmology department. Further studies with a larger sample size and other ethnic populations as well as patients enrolled from multiple sources are warranted to confirm our 
findings. Additionally, we only focused on eleven SNPs in the JAK-STAT pathway, and it is possible that other unknown SNPs might be associated with AAU risk.

In conclusion, this study reveals that genetic polymorphisms of JAK-STAT pathway genes, including JAK1, JAK2, STAT1, IRF1, and NOS2, may not be involved in susceptibility to AAU risk in the Han Chinese population.

\section{Competing Interests}

The authors declare that there is no conflict of interests regarding the publication of this paper.

\section{Authors' Contributions}

Ling Cheng and Hongsong Yu contributed equally to this work.

\section{Acknowledgments}

The authors would like to thank Professor Peizeng Yang for his great help in our study. They are also grateful to all donors enrolled in this study. This work was supported by the National Natural Science Foundation Project (81200678, 81670844), Fundamental and Advanced Research Program of Chongqing (cstc2015jcyjA10022), Science and Technology Project of Chongqing Municipal Education Commission (KJ1500236), Scientific Research Program of Science and Technology Commission of Yuzhong District of Chongqing (20150102), and National Key Clinical Specialties Construction Program of China.

\section{References}

[1] D.-K. Hwang, Y.-J. Chou, C.-Y. Pu, and P. Chou, "Epidemiology of uveitis among the Chinese population in Taiwan: A Population-Based Study," Ophthalmology, vol. 119, no. 11, pp. 2371-2376, 2012.

[2] K. Nakao and N. Ohba, "Prevalence of endogenous uveitis in Kagoshima Prefecture, Southwest Japan," Nippon Ganka Gakkai Zasshi, vol. 100, no. 2, pp. 150-155, 1996.

[3] D. C. Gritz and I. G. Wong, "Incidence and prevalence of uveitis in Northern California: the Northern California Epidemiology of Uveitis Study," Ophthalmology, vol. 111, no. 3, pp. 491-500, 2004.

[4] R. B. Nussenblatt and I. Gery, "Experimental autoimmune uveitis and its relationship to clinical ocular inflammatory disease," Journal of Autoimmunity, vol. 9, no. 5, pp. 575-585, 1996.

[5] P. Yang, Z. Zhang, H. Zhou et al., "Clinical patterns and characteristics of uveitis in a tertiary center for uveitis in China," Current Eye Research, vol. 30, no. 11, pp. 943-948, 2005.

[6] J. T. Gran and J. F. Skomsvoll, "The outcome of ankylosing spondylitis: a study of 100 patients," British Journal of Rheumatology, vol. 36, no. 7, pp. 766-771, 1997.

[7] L. P. Robertson and M. J. Davis, "A longitudinal study of disease activity and functional status in a hospital cohort of patients with ankylosing spondylitis," Rheumatology, vol. 43, no. 12, pp. 1565-1568, 2004.
[8] J. H. Chang, P. J. McCluskey, and D. Wakefield, "Acute anterior uveitis and HLA-B27," Survey of Ophthalmology, vol. 50, no. 4, pp. 364-388, 2005.

[9] M. Huhtinen and A. Karma, "HLA-B27 typing in the categorisation of uveitis in a HLA-B27 rich population," British Journal of Ophthalmology, vol. 84, no. 4, pp. 413-416, 2000.

[10] S. Torres, S. Borges, and A. Artiles, "HLA-B27 and clinical features of acute anterior uveitis in Cuba," Ocular Immunology and Inflammation, vol. 21, no. 2, pp. 119-123, 2013.

[11] R. Saari, R. Lahti, K. M. Saari et al., "Frequency of rheumatic diseases in patients with acute anterior uveitis," Scandinavian Journal of Rheumatology, vol. 11, no. 2, pp. 121-123, 1982.

[12] A. B. Beckingsale, J. Davies, J. M. Gibson, and A. Ralph Rosenthal, "Acute anterior uveitis, ankylosing spondylitis, back pain, and HLA-B27," British Journal of Ophthalmology, vol. 68, no. 10, pp. 741-745, 1984.

[13] G. P. Thomas and M. A. Brown, "Genetics and genomics of ankylosing spondylitis," Immunological Reviews, vol. 233, no. 1, pp. 162-180, 2010.

[14] P. C. Robinson and M. A. Brown, "Genetics of ankylosing spondylitis," Molecular Immunology, vol. 57, no. 1, pp. 2-11, 2014.

[15] C. Lopez-Larrea, K. Sujirachato, N. K. Mehra et al., "HLAB27 subtypes in Asian patients with ankylosing spondylitis. Evidence for new associations," Tissue Antigens, vol. 45, no. 3, pp. 169-176, 1995.

[16] P. C. Robinson, T. A. M. Claushuis, A. Cortes et al., "Genetic dissection of acute anterior uveitis reveals similarities and differences in associations observed with ankylosing spondylitis," Arthritis and Rheumatology, vol. 67, no. 1, pp. 140-151, 2015.

[17] H. Li, S. Hou, H. Yu et al., "Association of genetic variations in TNFSF15 with acute anterior uveitis in Chinese Han," Investigative Ophthalmology and Visual Science, vol. 56, no. 8, pp. 4605-4610, 2015.

[18] Q. Xiang, L. Chen, J. Fang et al., “TNF receptor-associated factor 5 gene confers genetic predisposition to acute anterior uveitis and pediatric uveitis," Arthritis Research and Therapy, vol. 15, no. 5, article R113, 2013.

[19] H. Yu, Y. Liu, L. Zhang et al., "FoxO1 gene confers genetic predisposition to acute anterior uveitis with ankylosing spondylitis," Investigative Ophthalmology \& Visual Science, vol. 55, no. 12, pp. 7970-7974, 2014.

[20] T. M. Martin, L. Bye, N. Modi et al., "Genotype analysis of polymorphisms in autoimmune susceptibility genes, CTLA-4 and PTPN22, in an Acute anterior uveitis cohort," Molecular Vision, vol. 15, pp. 208-212, 2009.

[21] Q. Zhang, J. Qi, S. Hou et al., "A functional variant of PTPN22 confers risk for Vogt-Koyanagi-Harada syndrome but not for ankylosing spondylitis," PLoS ONE, vol. 9, no. 5, article e96943, 2014.

[22] S.-S. Zhao, J.-W. Hu, J. Wang, X.-J. Lou, and L.-L. Zhou, "Inverse correlation between $\mathrm{CD} 4^{+} \mathrm{CD} 25^{\text {high }} \mathrm{CD} 127^{\text {low/- }}$ regulatory $\mathrm{T}$ cells and serum immunoglobulin $\mathrm{A}$ in patients with newonset ankylosing spondylitis," Journal of International Medical Research, vol. 39, no. 5, pp. 1968-1974, 2011.

[23] W. Zou, Z. Wu, X. Xiang, S. Sun, and J. Zhang, "The expression and significance of $\mathrm{T}$ helper cell subsets and regulatory $\mathrm{T}$ cells CD4+CD25+ in peripheral blood of patients with human leukocyte antigen B27-positive acute anterior uveitis," Graefe's Archive for Clinical and Experimental Ophthalmology, vol. 252, no. 4, pp. 665-672, 2014. 
[24] C. Wang, Q. Liao, Y. Hu, and D. Zhong, "T lymphocyte subset imbalances in patients contribute to ankylosing spondylitis," Experimental and Therapeutic Medicine, vol. 9, no. 1, pp. 250256, 2015.

[25] A. Tedgui and Z. Mallat, "Cytokines in atherosclerosis: pathogenic and regulatory pathways," Physiological Reviews, vol. 86, no. 2, pp. 515-581, 2006.

[26] P. J. Murray, “The JAK-STAT signaling pathway: Input and output integration," Journal of Immunology, vol. 178, no. 5, pp. 2623-2629, 2007.

[27] A. N. Mathur, H.-C. Chang, D. G. Zisoulis et al., "Stat3 and Stat4 direct development of IL-17-secreting Th cells," Journal of Immunology, vol. 178, no. 8, pp. 4901-4907, 2007.

[28] G. K. Hansson, "Regulation of immune mechanisms in atherosclerosis," Annals of the New York Academy of Sciences, vol. 947, pp. 157-165, 2001.

[29] T. Shea-Donohue, A. Fasano, A. Smith, and A. Zhao, "Enteric pathogens and gut function: role of cytokines and STATs," Gut Microbes, vol. 1, no. 5, pp. 316-324, 2010.

[30] T. Taniguchi, K. Ogasawara, A. Takaoka, and N. Tanaka, "IRF family of transcription factors as regulators of host defense," Annual Review of Immunology, vol. 19, pp. 623-655, 2001.

[31] M. Lohoff, D. Ferrick, H.-W. Mittrücker et al., "Interferon regulatory factor-1 is required for a $\mathrm{T}$ helper 1 immune response in vivo," Immunity, vol. 6, no. 6, pp. 681-689, 1997.

[32] S. Taki, T. Sato, K. Ogasawara et al., "Multistage regulation of Th1-type immune responses by the transcription factor IRF-1," Immunity, vol. 6, no. 6, pp. 673-679, 1997.

[33] C. Bogdan, "Nitric oxide and the immune response," Nature Immunology, vol. 2, no. 10, pp. 907-916, 2001.

[34] S. Brahmachari and K. Pahan, "Myelin basic protein priming reduces the expression of Foxp 3 in T cells via nitric oxide," The Journal of Immunology, vol. 184, no. 4, pp. 1799-1809, 2010.

[35] S.-W. Lee, H. Choi, S.-Y. Eun, S. Fukuyama, and M. Croft, "Nitric oxide modulates TGF- $\beta$-directive signals to suppress Foxp 3 + regulatory $\mathrm{T}$ cell differentiation and potentiate Th1 development," Journal of Immunology, vol. 186, no. 12, pp. 69726980, 2011.

[36] D. A. Jabs, R. B. Nussenblatt, J. T. Rosenbaum, and Standardization of Uveitis Nomenclature Working G, "Standardization of uveitis nomenclature for reporting clinical data. Results of the First International Workshop," American Journal of Ophthalmology, vol. 140, pp. 509-516, 2005.

[37] S. Van Der Linden, H. A. Valkenburg, and A. Cats, "Evaluation of diagnostic criteria for ankylosing spondylitis. A proposal for modification of the New York criteria," Arthritis and Rheumatism, vol. 27, no. 4, pp. 361-368, 1984.

[38] D. Ellinghaus, E. Ellinghaus, R. P. Nair et al., "Combined analysis of genome-wide association studies for Crohn disease and psoriasis identifies seven shared susceptibility loci," American Journal of Human Genetics, vol. 90, no. 4, pp. 636-647, 2012.

[39] G. Fortunato, G. Calcagno, V. Bresciamorra et al., "Multiple sclerosis and hepatitis $C$ virus infection are associated with single nucleotide polymorphisms in interferon pathway genes," Journal of Interferon and Cytokine Research, vol. 28, no. 3, pp. 141-152, 2008.

[40] W.-H. Hahn, J.-S. Suh, S. H. Cho, B.-S. Cho, and S.-D. Kim, "Polymorphisms of signal transducers and activators of transcription 1 and 4 (STAT1 and STAT4) contribute to progression of childhood IgA nephropathy," Cytokine, vol. 50, no. 1, pp. 6974, 2010.
[41] S. Hou, J. Qi, Q. Zhang et al., "Genetic variants in the JAK1 gene confer higher risk of Behcet's disease with ocular involvement in Han Chinese," Human Genetics, vol. 132, no. 9, pp. 1049-1058, 2013.

[42] K. Hu, S. Hou, F. Li, Q. Xiang, A. Kijlstra, and P. Yang, "JAK1, but not JAK2 and STAT3, confers susceptibility to Vogt-Koyanagi-Harada (VKH) syndrome in a Han Chinese population," Investigative Ophthalmology and Visual Science, vol. 54, no. 5, pp. 3360-3365, 2013.

[43] A. Cortes, J. Hadler, J. P. Pointon et al., "Identification of multiple risk variants for ankylosing spondylitis through highdensity genotyping of immune-related loci," Nature Genetics, vol. 45, no. 7, pp. 730-738, 2013.

[44] P. A. Shaiq, P. E. Stuart, A. Latif et al., "Genetic associations of psoriasis in a Pakistani population," British Journal of Dermatology, vol. 169, no. 2, pp. 406-411, 2013.

[45] S.-K. Yang, Y. Jung, H. Kim, M. Hong, B. D. Ye, and K. Song, "Association of FCGR2A, JAK2 or HNF4A variants with ulcerative colitis in Koreans," Digestive and Liver Disease, vol. 43, no. 11, pp. 856-861, 2011.

[46] M. García-Bermúdez, R. López-Mejías, F. Genre et al., "Lack of association between JAK3 gene polymorphisms and cardiovascular disease in Spanish patients with rheumatoid arthritis," BioMed Research International, vol. 2015, Article ID 318364, 11 pages, 2015.

[47] R. López-Mejías, M. García-Bermúdez, C. González-Juanatey et al., "NFKB1-94ATTG ins/del polymorphism (rs28362491) is associated with cardiovascular disease in patients with rheumatoid arthritis," Atherosclerosis, vol. 224, no. 2, pp. 426-429, 2012.

[48] H. J. Park, J. W. Kim, B.-S. Cho, and J.-H. Chung, "Association of $\mathrm{BH} 3$ interacting domain death agonist (BID) gene polymorphisms with proteinuria of immunoglobulin A nephropathy," Scandinavian Journal of Clinical and Laboratory Investigation, vol. 74, no. 4, pp. 329-335, 2014. 

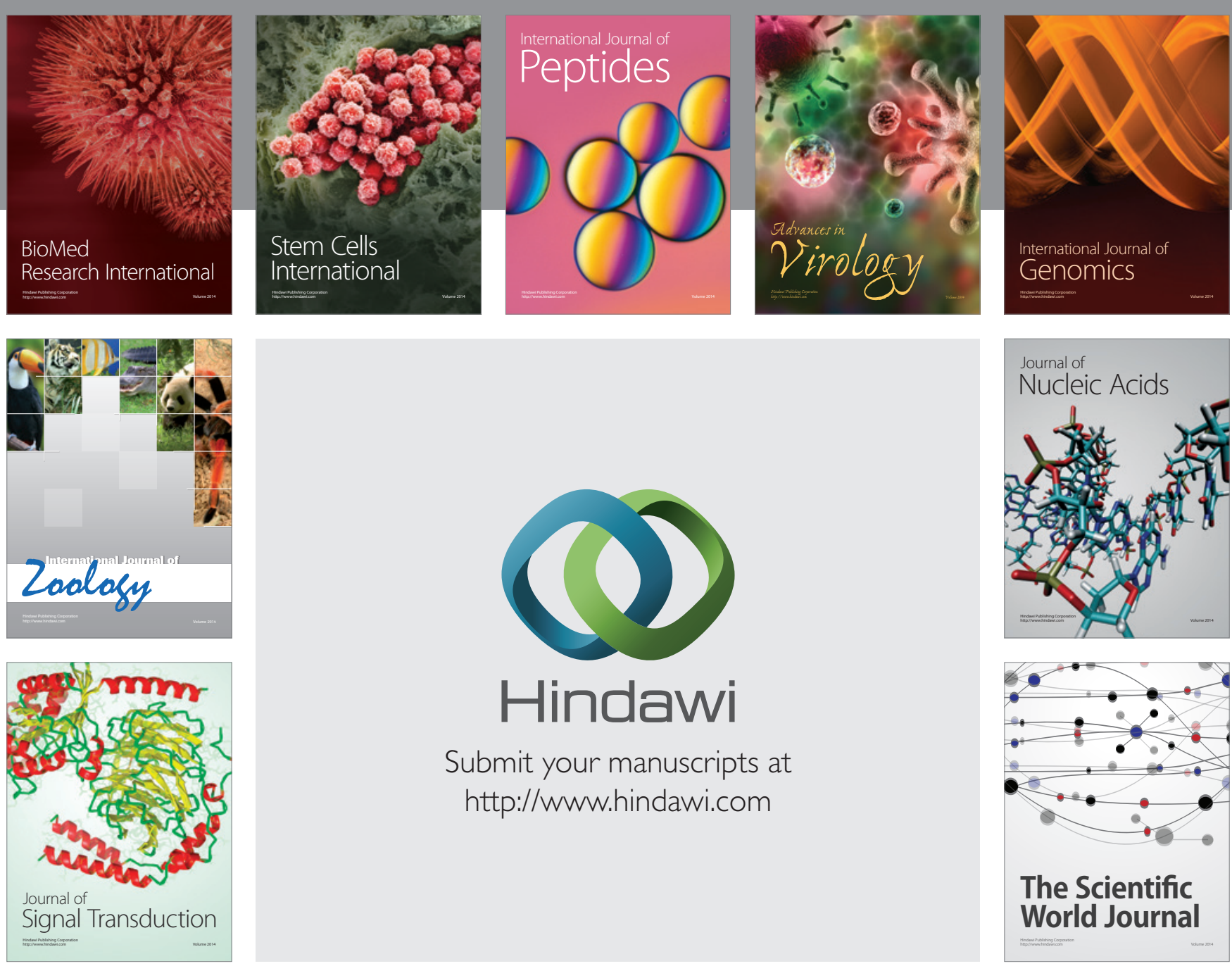

Submit your manuscripts at

http://www.hindawi.com
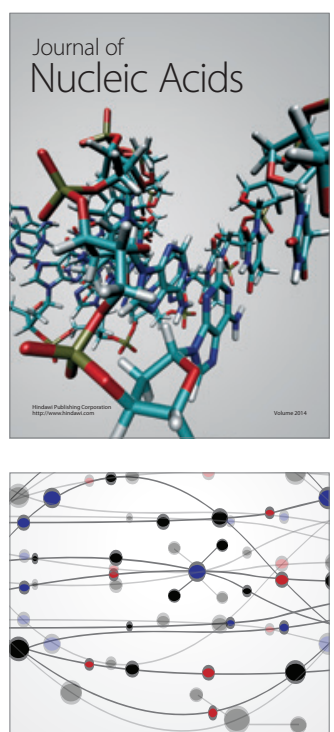

The Scientific World Journal
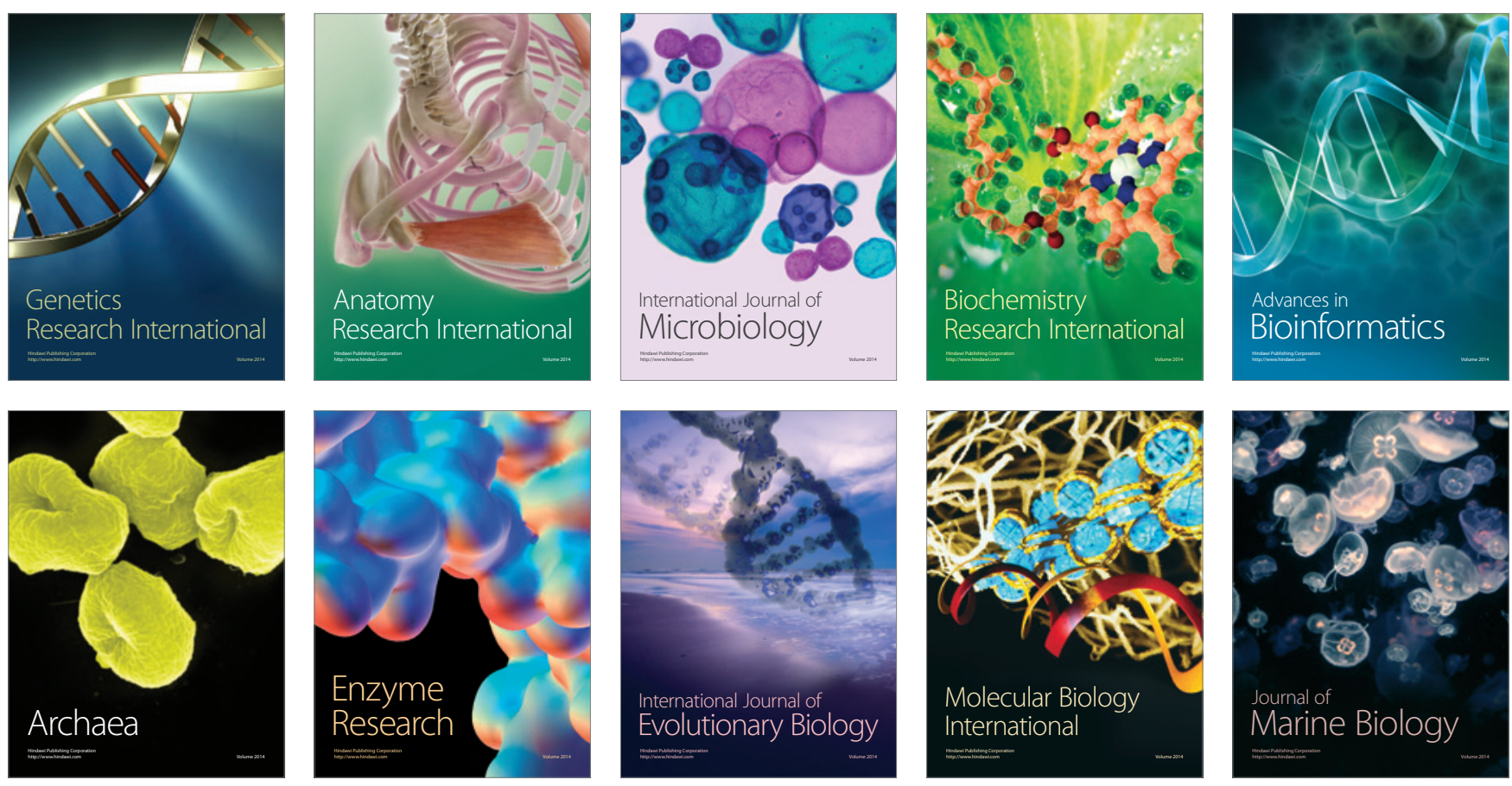